\title{
Authors for this volume
}

Gunnar Andersson, Stockholm University, Department of Sociology, Demography Unit, Stockholm, Sweden

Giovanna Boccuzzo, Department of Statistical Sciences, University of Padua, Padua, Italy

John Bongaarts, Population Council, New York, NY, USA

Nikolai Botev, United Nations Population Fund (UNFPA), Bratislava, Slovakia

William P. Butz, Population Reference Bureau, Washington, D.C., USA

Marcantonio Caltabiano, Department of Economics, Statistics, Mathematics and Sociology, University of Messina, Messina, Italy

Gianpiero Dalla Zuanna, Department of Statistical Sciences, University of Padua, Padua, Italy

Anne H. Gauthier, Department of Sociology, University of Calgary, Calgary, Alberta, Canada

Marzia Loghi, Istat - Italian National Statistical Institute, Rome, Italy

Marit Rønsen, Research Department, Statistics Norway, Oslo, Norway

Kari Skrede, Research Department, Statistics Norway, Oslo, Norway

Olivier Thévenon, French national institute for demographic studies (Institut national d'études démographiques, INED), Paris, France

Wolfgang Lutz, World Population Program, International Institute for Applied Systems Analysis, Laxenburg, Austria, and Vienna Institute of Demography, Austrian Academy of Sciences, Vienna, Austria

Ariane Pailhé, French national institute for demographic studies (Institut national d'études démographiques, INED), Paris, France

Dimiter Philipov, Vienna Institute of Demography, Austrian Academy of Sciences, Vienna, Austria

Clémentine Rossier, French national institute for demographic studies (Institut national d'études démographiques, INED), Paris, France

Ronald R. Rindfuss, East-West Center, Honolulu, HI, USA and Carolina Population Center, University of North Carolina, Chapel Hill, NC, USA

Sarah R. Brauner-Otto, Carolina Population Center, University of North Carolina, Chapel Hill, NC, USA

Laurent Toulemon, French national institute for demographic studies (Institut national d'études démographiques, INED), Paris, France 